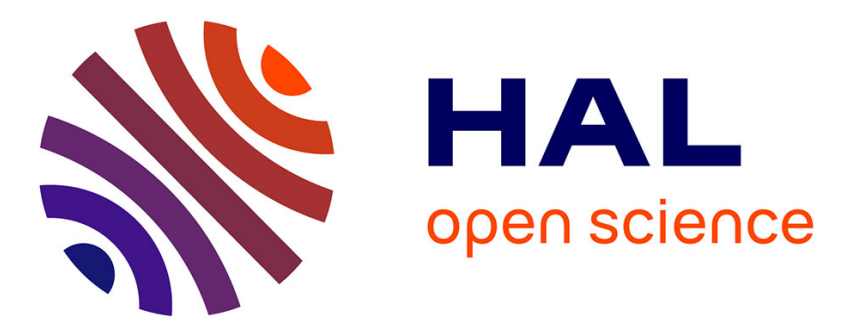

\title{
Coalescence in concentrated emulsions: theoretical predictions and comparison with experimental bottle test behaviour
}

Huy-Hong-Quan Dinh, Enric Santanach-Carreras, Véronique Schmitt, François Lequeux

\section{To cite this version:}

Huy-Hong-Quan Dinh, Enric Santanach-Carreras, Véronique Schmitt, François Lequeux. Coalescence in concentrated emulsions: theoretical predictions and comparison with experimental bottle test behaviour. Soft Matter, 2020, 16 (45), pp.10301-10309. 10.1039/d0sm01459a . hal-02972228

\author{
HAL Id: hal-02972228 \\ https://hal.science/hal-02972228
}

Submitted on 1 Oct 2021

HAL is a multi-disciplinary open access archive for the deposit and dissemination of scientific research documents, whether they are published or not. The documents may come from teaching and research institutions in France or abroad, or from public or private research centers.
L'archive ouverte pluridisciplinaire HAL, est destinée au dépôt et à la diffusion de documents scientifiques de niveau recherche, publiés ou non, émanant des établissements d'enseignement et de recherche français ou étrangers, des laboratoires publics ou privés. 


\title{
Journal Name
}

ARTICLE TYPE

Cite this: DOI: $00.0000 / x x x x x x x x x x$

Coalescence in concentrated emulsions: Theoretical predictions and comparison with experimental bottle test behaviour

\author{
Huy-Hong-Quan Dinh ${ }^{* a, b}$, Enric Santanach-Carreras ${ }^{a, b}$, Véronique Schmitt ${ }^{c}$ and François \\ Lequeux ${ }^{d, e}$
}

Received Date

Accepted Date

DOI: 00.0000/ $x x x x x x x x x x$

Fusion between emulsion drops, also called coalescence, may be undesirable for storage or sought after depending on the desired application. In this latter case, a complete separation of the two liquids composing the emulsion is required. The same objective may be aimed in foams. We have performed bottle test experiments on a model system of water in oil (w/o) emulsion stabilized by high amount of the hydrophobic surfactant Span 80. We observe two regimes for emulsion separation: after the first regime which is fast and includes sedimentation of the water droplets, the second regime exhibits a very dense and stable emulsion zone. We predict the initial thickness of the dense zone as a simple function of surfactant concentration and mean droplet size. From the assumption that the coalescence rate depends only on the area of the thin film between two contacted droplets, we quantitatively model the separation kinetics of the dense emulsion zone. Our results give rise to a simple method that allows measuring the coalescence frequency per unit area, only by monitoring bottle test experiments.

\section{Introduction}

Emulsion is a mixture of two or more immiscible liquids. Most of the emulsion systems are often formed by blending an oily phase with an aqueous one, in addition with the surface-active agents to enhance their kinetic stability. The study of emulsion stability plays a crucial role in many realms of technology such as foods, cosmetics or petroleum engineering, which explains why numerous studies have been devoted to this subject. The life time of emulsions could widely vary from minutes to months depending on the emulsion type. Emulsion destabilisation occurs via different mechanisms such as sedimentation/creaming responsible for a phase separation between the emulsion and the continuous phase or as Ostwald ripening and coalescence responsible for emulsion destruction and a phase separation between the continuous and dispersed phases ${ }^{1}$. Whereas the first two processes are well described in literature, the understanding of coalescence, which is the major factor of the emulsion destabilisation, remains highly debatable. In the absence of surfactant, the coalescence

\footnotetext{
${ }^{a}$ Laboratoire Physico-Chimie des Interfaces Complexes, Bâtiment CHEMSTARTUP, RD 817, 64170 Lacq, France. Email: huy-hong-quan.dinh@total.com

${ }^{b}$ TOTAL S.A., Pôle d'Etudes et de Recherches de Lacq, BP 47, 64170 Lacq, France

c Centre de Recherche Paul Pascal, 115 Avenue Schweitzer, 33600 Pessac, France

d Laboratoire Sciences et Ingénierie de la Matière Molle, ESPCI Paris, PSL University, Sorbonne Université, CNRS UMR 7615, F-75005 Paris, France

${ }^{e}$ Laboratoire Physico-Chimie des Interfaces Complexes, ESPCI Paris, 10 rue Vauquelin, F-75231 Paris, France

Electronic Supplementary Information (ESI) available: See DOI: 00.0000/00000000.
}

is fast and limited only by hydrodynamics, and occurs as soon as the film of one phase, sandwiched in between two droplets of the other phase, decreases to a thickness of a few tens of nanometers where Van der Waal's attraction leads to the piercing of the film 2 [3. However, in the presence of surfactants the situation is more confuse. Several scenarios have been suggested to describe the coalescence mechanism of thin films in presence of surfactants. The first one consists of the competition between capillary pressure and disjoining pressure ${ }^{45}$; the thin film bursts when the capillary pressure exceeds the disjoining one. However, no direct proof of this effect has been reported to the best of our knowledge. Moreover, this argument omits the role of surface elasticity, which has been confirmed in most of the recent experimental works 6 . Indeed, the nature of surfactant is critical for the film stability 9 . The coalescence is likely thermally activated as suggested by De Vries 10 . This leads to the hypothesis that the coalescence events are purely statistical, in which the probability of coalescence is - for a given surfactant at a given concentration - proportional to the thin film area 11 , as confirmed recently in $2 \mathrm{~d}$ emulsions 14 . The proportionality coefficient $\omega$ (namely the coalescence frequency per unit area), which accounts for the physico-chemistry of the studied system, is thus the key parameter characterizing the whole coalescence process.

Classical bottle test consists in mixing oil and water in presence of surfactant and measuring the rate of phase separation. In a bottle test, two successive regimes of emulsion separation can be observed. The first regime corresponds to a fast, buoyancy- 
driven separation with simultaneously fast coalescence; the second regime exhibits a dense and stable zone of emulsion, which is not easily destructed even by centrifugation 15 . However, a quantitative relation between the thickness of the dense zone and the surfactant concentrations, has not been established yet. Furthermore, the study of separation kinetics remains likely either at the first regime level, or in the hydrodynamic contexts without presence of surfactants ${ }^{18} 19$. The separation kinetics of the dense zone per se, in which the droplets are fully covered by surfactants, remains largely unknown.

In this paper, we bring a fresh eye for the study of coalescence behaviour and of the characterisation of emulsion stabilized by surfactants, through a classical and simple bottle test method. We give evidence that the coalescence probability depending on thin film area could greatly explain the observations from our bottle test experiments. The latter leads us to introduce a simple and inexpensive method to determine the coalescence frequency per unit area $\omega$ of the system. A complete model for separation kinetics of the dense emulsion zone will be proposed. In parallel with the kinetics study, we directly model the amount of the generated dense zone volume as function of surfactant concentrations.

\section{Experimental section}

\subsection{Emulsion preparation}

The oil phase of the emulsions consists of dodecane (Thermo Fisher Scientific- Ref. 117590025) to which we add various concentrations of Span 80 (Fisher Scientific - Ref. 15474919) to favor the formation of water-in-oil emulsions. Sodium Chloride (Fisher Scientific) is added to deionized water (18 $\mathrm{M} \Omega$ ) for the aqueous phase; to ensure the robustness of our results, we have performed tests at $10 \mathrm{mM}$ and $100 \mathrm{mM}$. The role of $\mathrm{NaCl}$ is two-fold: it allows screening all possible electrostatic interactions that may be due to traces of ionic surfactants ${ }^{20}$ and preventing Ostwald ripening to be the prevalent destabilisation mechanism by even further reducing the dispersed phase solubility in the continuous phase.

For all bottle tests presented hereinafter, unless otherwise specified, we fix the water/oil volume ratio to $1: 1$ and the total volume of fluids to $15 \mathrm{ml}$. The vials used have diameter of approximately $2 \mathrm{~cm}$ and $24 \mathrm{ml}$ volume. First, we add $7.5 \mathrm{ml}$ of water and then we complete with $7.5 \mathrm{ml}$ of oily phase. Since the solubility of water in dodecane, and vice versa, is finite but not null, we let the fluids equilibrate at rest for 30 minutes before proceeding with the emulsification step.

Droplet size and size distribution are key parameters in emulsions. For this reason, and to add soundness to our model, we prepare emulsions with either a Vortexer (IKA-MS1), working at $2500 \mathrm{rpm}$, and an Ultra-Turrax (IKA-T10) which is used with rotational speeds ranging from $10000 \mathrm{rpm}$ up to $30000 \mathrm{rpm}$.

\subsection{Interfacial tension measurement}

The stability of an emulsion greatly depends on the available amount of surfactant. The type of surfactants present; their amounts, absolute and relative to each other, and the induced decrease in interfacial tension, will also play a critical role on the initial state of the emulsion morphology: size of droplets, sta- bility. The system under study in terms of interfacial tension for the whole range of surfactant concentrations covered, is therefore of upmost importance. To measure the water/oil interfacial tension, we use a commercial set-up (TECLIS) based on the pendant drop method: we create a water droplet, a few $\mu 1$ in volume, in a bath of Dodecane containing the desired Span 80 concentration. The software finds the best fit to the curvature of droplet interface using $\gamma$ as the adjusting parameter. To avoid artifacts due to mass transfer between the different phases, we contact the aqueous and oil phases for at least 24 hours prior to measuring the interfacial tension for each condition in order to equilibrate the partition of the surfactants between oil and water. By varying the concentration of surfactant in the bulk, we construct the isotherm curve for our system, which is used in our theoretical models. The experimental data is presented in the SI.

\subsection{Image acquisition and image treatment}

To quantify emulsion stability, we follow the temporal evolution of two interfaces: the emulsion/water interface (at the bottom of the vial), which creates the water leg, and the oil/emulsion interface (at the top of the vial) due to the gravity-driven settling of water droplets induced by the density difference between oil and water. The positions of these interfaces from the bottom of the vial are noted as $H_{w}$ and $H_{S}$ respectively and are shown in Figure 1 A (right). We take still pictures at regular time intervals of the vials using a Nikon D5100 camera and backlighting to capture the position of these two interfaces at different stages of the destabilisation process Figure 13 .

From each photography, we extract the position of both oil/emulsion $\left(H_{S}\right)$ and emulsion/water $\left(H_{w}\right)$ interfaces using a Matlab program. The interfaces are not necessarily straight and this can induce significant noise in the determination of the interface position. This is particularly true for the $H_{w}$ interface where a meniscus is clearly observed (Figure $1 \mathrm{~B}$ ). For this reason, we average the intensity of the image about the $\mathrm{x}$-direction and look at the intensity profile of this average along the direction of the vial height. The noise in the $H_{S}$ interface, basically in the order of 1 droplet radius, is much smaller. Taking the derivative of this averaged intensity profile allows then to readily extract the position of both interfaces.

\section{Results and discussion}

The process of emulsion separation and destabilisation is a complex one where both $H_{S}$ and $H_{w}$ interfaces evolve with time. An example is reported in Figure 1 for an emulsion stabilized by 800 ppm of Span 80 with $10 \mathrm{mM}$ of $\mathrm{NaCl}$ in aqueous phase. For each of these interfaces, at least two regimes can be identified. In the case of $H_{S}$, individual water droplets settle fast because of the density difference of water with dodecane until the volume fraction of dispersed phase reaches $\sim 0.8$. This dense emulsion is then compressed at a much slower rate via the drainage of the interstitial oil present between the water droplets. An interplay between the weight of the emulsion and the permeability of this dynamic porous media dictates the compression kinetics and leads to a volume fraction gradient within this dense emulsion. The char- 
acteristic time scale of the settling is in the order of minutes and hence $H_{S}$ position can be considered as being constant throughout the irreversible destabilisation process described hereinafter; This is clearly seen in the red curve in Figure $1 \mathrm{~h}$. It is important to notice that the position of the $H_{S}$ interface is not indicative of the stability of the emulsion and hence we will not further discuss it in this work. We will focus on the evolution of the $H_{w}$ interface whose evolution is indicative of irreversible changes in the system through coalescence.

\subsection{Emulsion characterisation: Observation of a dense zone and the existence of two regimes of separation}

While observing the evolution of the $H_{w}$ interface in water/oil emulsions formed with high concentrations of Span 80, we clearly identify 2 different regimes (Figure $1 \mathrm{C}$ ). A first regime, of characteristic time $10^{1}-10^{2}$ minutes, during which the emulsion separates rapidly. This regime is indeed due to the presence of surfactant-unsaturated interfaces in the freshly made emulsion. In order to increase surface concentration of surfactant molecules, the emulsion naturally reduces its amount of surface through coalescence events. We will not focus on the kinetics of this first regime, as it represents no more than 0.01 of the whole destabilisation process, which can last up to $10^{5}$ minutes, id est 2 months, as Figure $1 \mathrm{C}$ shows. In the second regime, the separation speed is significantly slow compared to the first one. The emulsion is very dense, average volume fraction of 0.8-0.9, with initially small and compact dispersed droplets. The position of the two interfaces are nearly flat, but we can observe that some of the droplets become very large, an irrefutable prove of the occurrence of coalescence events within the dense emulsion zone, as seen in Figure 13 (III and IV in particular).

This dense zone is not easily destructed by centrifugation, even at high accelerations. We claim that the dense zone is completely thermally activated, in which the key parameter for the stability is the coalescence frequency per unit area $\omega$ between the emulsion droplets 21 . This parameter depends on the lifetime and the critical thickness of the thin film between 2 droplets undergoing coalescence. The presence of surfactants like Span 80 on the droplets surface reinforces the thin-film lifetime and hence increases emulsion stability.

The transition between the two regimes occurs at a time $\tau^{\star}$ and at a position of the free-water interface that we call, $H_{w}^{\star}$. Beyond this point, we observe a quasi-plateau where the height of free water interface $H_{w}$ increases very slowly (Figure 1 $\mathrm{C}$ ) and whose length is described by the characteristic time, $\tau_{C}$. After this quasiplateau, the separation kinetics seem to accelerate. Experiments carried out by varying the concentrations of Span 80 show that the quasi-plateau position, $H_{w}^{\star}$, correlates with surfactant concentration $c_{0}$ in the oil phase.

Considering the partition of Span 80 molecules between droplet surfaces and the liquid bulk phases, we explain and provide a theoretical approach of this observation. One expects to improve emulsion stability with an increasing concentration of Span 80 molecules per unit droplet area. Moreover, we may as-

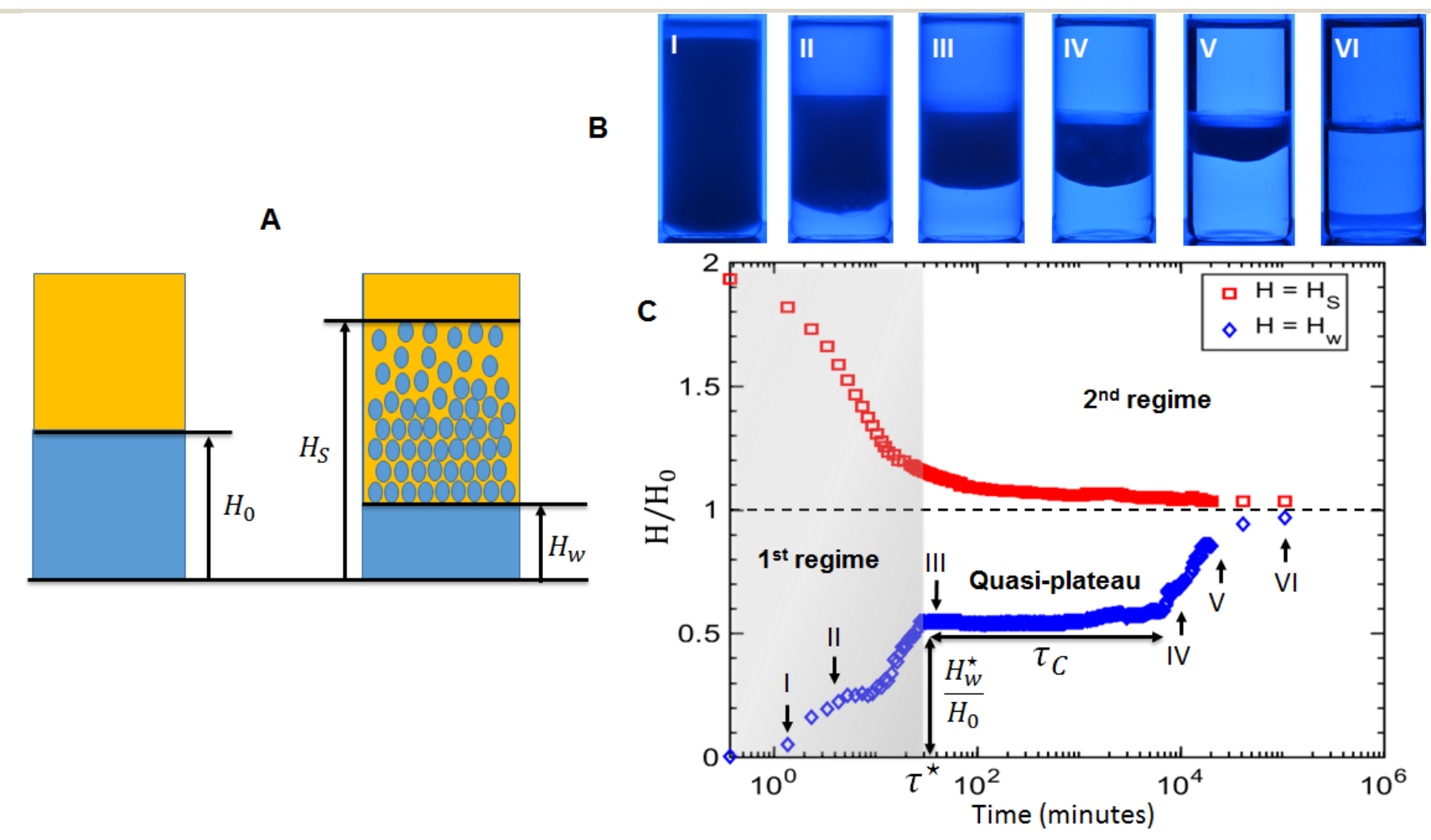

Fig. 1 (A) Definition of interfaces position measured in bottle test (B) Snapshots of bottle test photos during emulsion destabilisation (C) Interfaces detected in bottle test: Sedimentation (Red) and Water-leg interface (Blue) 
sume that the emulsion becomes stable, and coalescence is significantly hindered, when the droplets surface are covered by 80 $90 \%$ of surfactant molecules 22 . This condition will be satisfied when the surfactant concentration in the bulk reaches a certain value $c^{\star}$. Experimentally speaking, this is the minimum concentration of Span 80 in oil needed to generate the dense zone. Below this concentration, no dense zone will be observed. We find that $c^{\star}$ takes value around $20 \mathrm{ppm}$ for our model system with Span 80. It corresponds to a surface concentration $\Gamma^{\star} \approx 0.8 \Gamma_{\text {sat }}$. The surface concentration $\Gamma^{\star}$ at the transition point of 2 regimes is calculated directly by a simple surfactant balance from the total concentration $c_{0}$ of Span 80 added in the oil phase prior to emulsification:

$$
\Gamma^{\star}=\frac{V_{0}}{N^{\star} 4 \pi R_{0}^{2}}\left(c_{0}-c^{\star}\right)
$$

Where $N^{\star}$ is the number of droplets, $R_{0}$ is mean droplet radius at the beginning of the second regime, $V_{0}$ is the total oil volume. The conservation of water volume gives:

$$
N^{\star} \frac{4 \pi R_{0}^{3}}{3}+H_{W}^{\star} S=H_{0} S=\frac{\Phi}{1-\Phi} V_{0}
$$

Where $H_{0}$ is the height of water column when the both liquids are completely separated, $S$ is the cross section of the tube and $\Phi$ is the volume fraction of water in our system (we focus on $\Phi=50 \%$ in most of the bottle tests). Combining equations (1) and (2), we deduce an expression for $H_{W}^{\star}$ :

$$
\frac{H_{W}^{\star}}{H_{0}}=1-\frac{1-\Phi}{\Phi} \frac{R_{0}}{3 \Gamma^{\star}}\left(c_{0}-c^{\star}\right)
$$

Equation (3) shows a linear relation between the quasi-plateau position $H_{w}^{\star}$, concentration of Span 80 , and $R_{0}$. To validate our explanation, we perform experiments at $\Phi=50 \%$ by varying concentrations of Span 80, $c_{0}$, and different mixing protocols that we expect to result in different mean droplet radii $R_{0}$. The outcome of each experiment is $H_{w}^{\star}$, which can be easily measured from the images. Our experimental measures obtained with emulsions prepared with and Ultra Turrax at 3 different rotational speeds, 11 000, 15000 and 30000 RPM; as well as a Vortexer, indeed show a linear relation between $H_{w}^{\star}$ and $c_{0}$ as can be seen in figure 2 . Interestingly, this implies a fixed $R_{0}$ for a given mixing protocol.

The solid lines represent the best fit to our experimental data obtained by fixing $c^{\star}=20 \mathrm{ppm}$ and $\Gamma^{\star}=0.8 \Gamma_{\text {sat }}$, with $\Gamma_{\text {sat }}=$ $5 \times 10^{-6} \mathrm{~mol} / \mathrm{m}^{2}$. The latter is calculated directly from our interfacial tension measurements (see SI for more details). From the slope of these fits, we extract a typical value of $R_{0}$ for each mixing protocol, which is the only remaining unknown parameter. Not surprisingly, increasing the mixing speed, and hence the energy input to emulsify the system, results in smaller droplets.

Note that the droplets radius $R_{0}$ at the beginning of the second regime might be slightly different at different concentrations of Span 80, even for the same mixing protocol. However the overall ranges of variation of $H_{w}^{\star}$ at different concentrations of Span 80 is still correlated to the concentrations of Span 80. Equation (3) gives an estimation of droplets radius range for the 3 levels of Ultra Turrax, which are: $R_{0}=5-7 \mu \mathrm{m}$ (30 $000 \mathrm{RPM}$ ), $R_{0}=8-10 \mu \mathrm{m}(15000 \mathrm{RPM})$ and $R_{0}=11-20 \mu \mathrm{m}$ (11 $\left.000 \mathrm{RPM}\right)$.

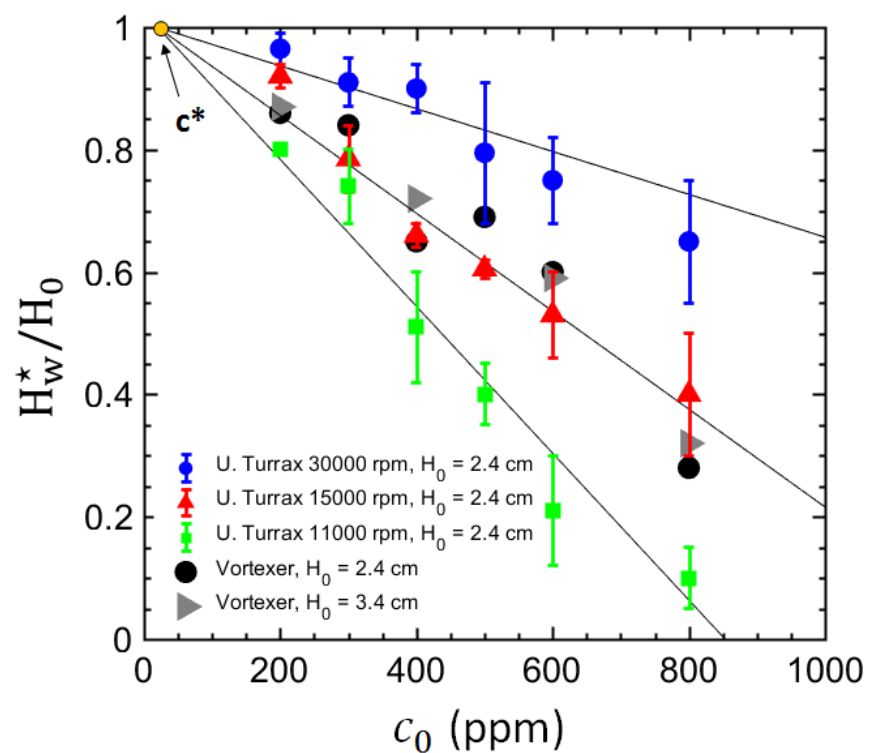

Fig. 2 Measure of $H_{w}^{*}$ for different concentrations of Span 80, and at different mixing protocols (Ultra Turrax at 11000, 15000, 30000 RPM and Vortexer at 2500 RPM)

Optical microscopy images of the formed emulsions confirm the radii predicted with our model (See SI). This result allows us to estimate the initial mean droplet radius within the dense zone at the regime transition, by only knowing the plateau position $H_{w}^{\star}$ and the surfactant concentration $c_{0}$.

Another way of confronting experiments to our model described by equation (3), is to vary $\Phi$ keeping the same mixing protocol. According to our model, the experimental plots of $H_{w}^{\star} / H_{0}$ versus $(1-\Phi) c_{0} / \Phi$ should be unified in a straight line, and this is, indeed, what our experimental data shows as seen in Figure 3. We note on this figure that when the concentration of surfac-

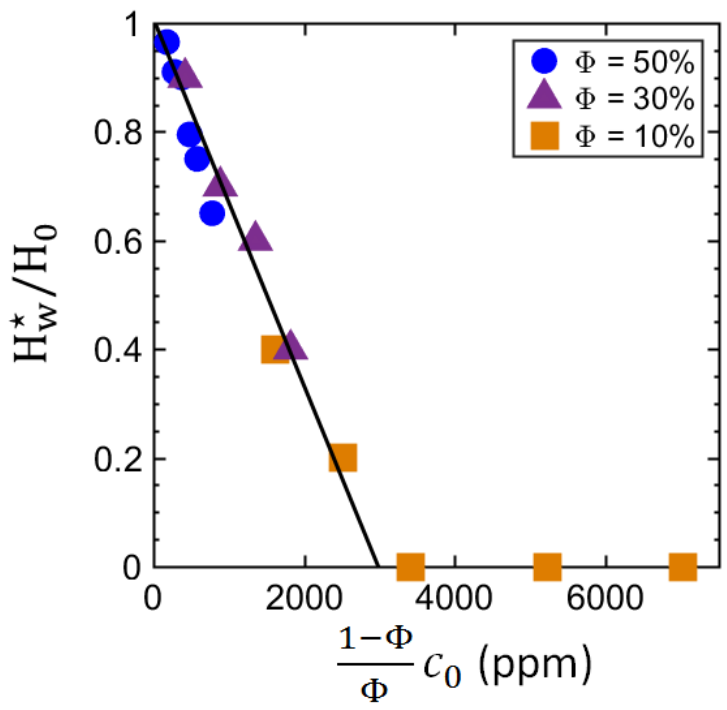

Fig. 3 Measure of $H_{w}^{*}$ at different water volume fraction $\Phi$, at mixing protocol of Ultra Turrax 30000 RPM 
tant, normalized by the volume ratio of the two phases, exceeds a given value, the thickness of the dense zone predicted by our simple model exceeds the thickness of the bottle. Physically, this means that the amount of surfactant present is large enough to cover all the interfaces of the emulsion, and thus the emulsion is already in the second regime at the end of its preparation.

\subsection{Modeling the duration of the quasi-plateau}

The separation time of our concentrated emulsion is very long; for some of the Span 80 concentration conditions tested, separation of $90 \%$ of the water volume could take more than $10^{5}$ minutes. Comparatively speaking, the first regime, which lasts only about 100 minutes, can be considered as fast. For this reason, we focus on the separation kinetics of the dense emulsion zone in the second regime. In particular, we concentrate on the duration of the quasi-plateau since, as we will show, it can give valuable and interesting insight on the coalescence of droplets within the dense zone.

In our context, we neglect the effect of Ostwald ripening because of the presence of osmotic pressure that inhibits mass transfer between the droplets. Therefore, droplet coalescence is the main factor for the destabilization of the dense emulsion zone. Our main hypothesis is that the probability of coalescence between two droplets $i$ and $j$ in contact is equal to the product of the coalescence rate per surface unit, $\omega$ with the area of the contact thin film $S_{i j}$. This relation is the bridge from the microscopic scale to the macroscopic one. For the sake of simplicity, we assume that $\omega$ is fixed during the whole separation process.

We call $\mathrm{z}$ the depth counted from the upper interface of emulsion, i.e. with excess oil phase. The height of this interface counted from the bottom is close to $H_{0}$ because the volume fraction of the dense zone is very high, as can be seen in Figure 13 and we will for the sake of simplicity assume that the water volume fraction of the dense zone can be approximated by 1 .

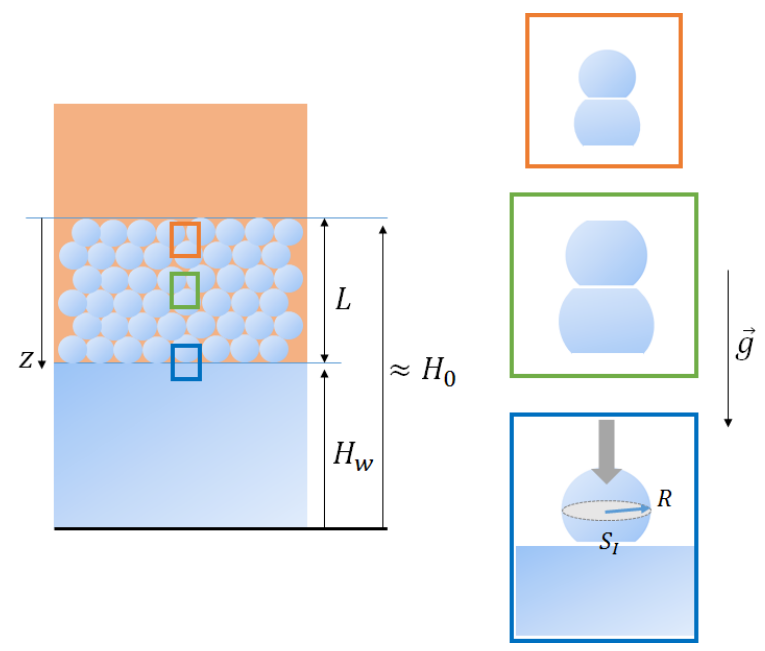

Fig. 4 Schema of dense zone and indications of lengths used in the model

The first ingredient in our model is the contact surface $S_{i j}$ between two droplets of size $R_{i}$ and $R_{j}$ at the depth $\mathrm{z}$ within the bulk of dense zone. Since the droplets are highly closed packed, the droplet at a depth $\mathrm{z}$ feels a hydrostatic pressure $\Delta P \approx \Delta \rho g z, 23$ where $\Delta \rho$ is the density difference between the aqueous and oil phases. Consider a small volume $\mathrm{V}$ at a depth $\mathrm{z}$, the contact of droplets within this volume yields the average stress tensor at the droplets surface over the volume V by the Irwin Kirkwood tensor:

$$
\hat{\sigma}=\frac{1}{V} \sum_{i, j \text { contact }} \vec{f}_{i j} \bigotimes \overrightarrow{r_{i j}}
$$

where $\vec{f}_{i j}$ is the contact force between droplets $\mathrm{i}$ and $\mathrm{j}, \overrightarrow{r_{i j}}$ is the connected vector between these two droplets. The normal component of this tensor is equivalent to the hydrostatic pressure, hence:

$$
\Delta P=\frac{1}{3} \operatorname{Tr}(\hat{\sigma})
$$

The contact force between droplets is induced by the pressure in the contact film. It has been established that the latter is simply a Laplace pressure $\gamma\left(\frac{1}{R_{i}}+\frac{1}{R_{j}}\right) \cdot \frac{24|25|}{2}$ This allows to explicit the pressure from equation (4). The pressure thus writes :

$$
\Delta P=\frac{1}{3 V} \sum n_{i j} \gamma\left(\frac{1}{R_{i}}+\frac{1}{R_{j}}\right) S_{i j}\left(R_{i}+R_{j}\right)
$$

Where $n_{i j}$ is the number of contacts between droplets $\mathrm{i}$ and $\mathrm{j}$ in volume $\mathrm{V}, \gamma$ is the water/oil interfacial tension, $S_{i j}$ is the thin film area between these droplets. The average over all the possible droplets in contact $n_{i j}$ can be estimated as follows. Consider a droplet $i$. It has a probability $p\left(R_{i}\right)$ to have a radius $R_{i}$. It occupies a volume $4 / 3 \pi R_{i}^{3}$. Let also $g_{c}\left(R_{j}, R_{i}\right)$ be the probability for the droplet $i$ to have a contact with droplet $j$ of radius $R_{j}$. The expression has also to be divided by two because each contact force is counted twice, from $i, j$ and $j, i$. This leads to the expression

$$
\Delta P=\sum_{i} p\left(R_{i}\right) \sum_{j=1} g_{c}\left(R_{j}, R_{i}\right) \frac{1}{8 \pi R_{i}^{3}} \gamma\left(\frac{1}{R_{i}}+\frac{1}{R_{j}}\right) S_{i j}\left(R_{i}+R_{j}\right)
$$

The sum over $j$ has to be done over the all neighboring droplets $j$ in contact with droplet $i$ - that may depend on the relative radius of droplets $i$ and its neighbors as detailed in the Appendix A. Due to polydispersity and droplets deformations, performing a summation of the previous equation is extremely tough. We will thus neglect the effect of polydispersity. For that, we will simply assume that all the droplets have the average droplet radius that we call $R$. This will allow to get the right scaling, under the assumption that the polydispersity does not evolve too much during coalescence. In fact, as shown in the Appendix A we expect the polydispersity evolution to be weak because the fastest growth in a mixture is expected to be the one of the average radius droplets. Finally, we assume that each droplet $i$ is surrounded by 12 other droplets. Under these approximations, the previous expression reduces to:

$$
\Delta P=\gamma S_{i j} \frac{6}{\pi R^{3}}
$$

This expression allows us to evaluate the contact area between droplets at a depth $z$, expliciting the pressure field:

$$
S_{i j}=\frac{\pi R^{3} \Delta \rho g z}{6 \gamma}
$$


Note that the contact area $S_{i j}(R)=\frac{\pi \Delta \rho g z}{6 \gamma} R^{3}$ has to remain smaller than $1 / 12$ of a droplet's total surface which is of the order of $4 \pi R^{2}$. Thus, expression (9) remains valid as long as $R$ is smaller than $R_{L} \approx 2 \gamma / \Delta \rho g z$. In our system, $z \sim 1 \mathrm{~cm}$ then $R_{L} \approx 330 \mu \mathrm{m}$. This radius is indeed much larger than the initial mean radius within the dense zone, which takes a value around $5-10 \mu \mathrm{m}$.

We now can estimate the droplets growth rate. Continuing with our assumption that the average size can be substituted to the size of individual droplets, we can write the average increase of volume of individual droplets. During each event of coalescence, the droplets have a volume that increases by the quantity $4 / 3 \pi R^{3}$. So if we follow a given droplet $i$, its volume will increase with time as following:

$$
\frac{d}{d t}\left(\frac{4}{3} \pi R^{3}\right) \approx 12 \omega S_{i j}\left(\frac{4}{3} \pi R^{3}\right)
$$

Combining equations (9) and (10) we get:

$$
\frac{d R}{d t} \approx \frac{2 \pi \omega \Delta \rho g z}{3 \gamma} R^{4}
$$

We remark that the growth rate of the droplets is proportional to the depth $\mathrm{z}$, hence the droplets which are close to the water/emulsion interface are generally bigger than the upper ones. Intergration of equation (11) writes:

$$
\frac{1}{R_{0}^{3}}-\frac{1}{R(z, t)^{3}}=\frac{2 \pi \omega \Delta \rho g z}{\gamma} t
$$

So the droplets radius diverges in a time $\tau_{C}$ that depends on depth $z$ and that is given by :

$$
\tau_{C}(z)=\frac{\gamma}{2 \pi \omega \Delta \rho g R_{0}^{3} z}
$$

The divergence time for the droplets which are close to the interface between emulsion and water homophase $\left(z \approx H_{0}-H_{w}^{\star}\right)$ is thus:

$$
\tau_{C}=\frac{\gamma}{2 \pi \omega \Delta \rho g R_{0}^{3} L_{0}}
$$

where $L_{0}=H_{0}-H_{w}^{\star}$ is the initial thickness of the dense zone (at the beginning of the second regime). An important remark from equation (12) is that the time evolution for the droplets at a depth $\mathrm{z}$ is very sharp. The radius grows very slowly for $t<\tau_{C}$, but diverges when $t=\tau_{C}$. This behavior allows to understand the separation kinetics shown in Figure $1 \mathrm{C}$. At the beginning of the second regime, the separation is very slow (the quasi-plateau) until a sharp increases of $H_{w}$ appears. We claim that this characteristic time, $\tau_{C}$, corresponds to the duration of the quasi-plateau. This duration $\tau_{C}$ is the lapse of time during which the droplets slowly coarsen through coalescence events before being large enough to exhibit a very fast coalescence. So from duration of the quasiplateau of the second regime $\tau_{C}$, we can deduce the coalescence rate $\omega$ :

$$
\omega=\frac{\gamma}{2 \pi \tau_{C} \Delta \rho g R_{0}^{3} L_{0}}
$$

We have seen that in the section 3.1 the initial average size of droplets in dense zone is related to the initial thickness of the dense zone by the equation (3):

$$
R_{0} \approx \frac{3 \Gamma_{\text {sat }}}{c_{0}} \frac{L_{0}}{H_{0}}
$$

Substituting this expression of $R_{0}$ in the equation 14 we get,

$$
\tau_{C} H_{0}=\frac{\gamma c_{0}^{3}}{54 \pi \omega \Delta \rho g \Gamma_{\text {sat }}^{3}}\left(\frac{L_{0}}{H_{0}}\right)^{-4}
$$

Under the assumption where $\omega$ is fixed for a given concentration $c_{0}$ of Span 80 within the dense zone, the plot of $\tau_{C} H_{0} / c_{0}^{3}$ as function of $L_{0} / H_{0}$ in a log-log scale is expected to have a slope of exponent -4 . Indeed, we plot this relation of different bottle tests at $800 \mathrm{ppm}$ and $1000 \mathrm{ppm}$ of Span 80 that we have performed, the slope of the best fit line is very close to -4 as Figure 5 shows. From a best fit of our experimental data, we estimate $\omega \approx 2 \times 10^{5}\left(1 / \mathrm{sm}^{2}\right)$, whose order of magnitude is in agreement with the ones measured on other systems 11 . This scaling law between $\tau_{C} H_{0}$ and $L_{0} / H_{0}$ remains valid as long as $H_{w}^{\star}>0$ or $L_{0}<H_{0}$. In the case of $H_{w}^{\star}=0$, one expects from equation (14) that $\tau_{C}$ is rather scaling with $1 / R_{0}^{3}$. Note that only a limited reduction in $\tau_{C}$ can be achieved by increasing the acceleration $g$ or height of liquid column $H_{0}$ because the maximum available contact surface, $S_{i j}$ in equation (9) is limited by the droplet's total area as previously discussed. Given the characteristic droplet size and interfacial tension of our model system, an increase of $g$ (or $H_{0}$ ) by a factor of 4 will be sufficient to reach this maximum amount of contact surface $S_{i j}$ This explains why the dense zone is not likely to be rapidly destructed by centrifugation.

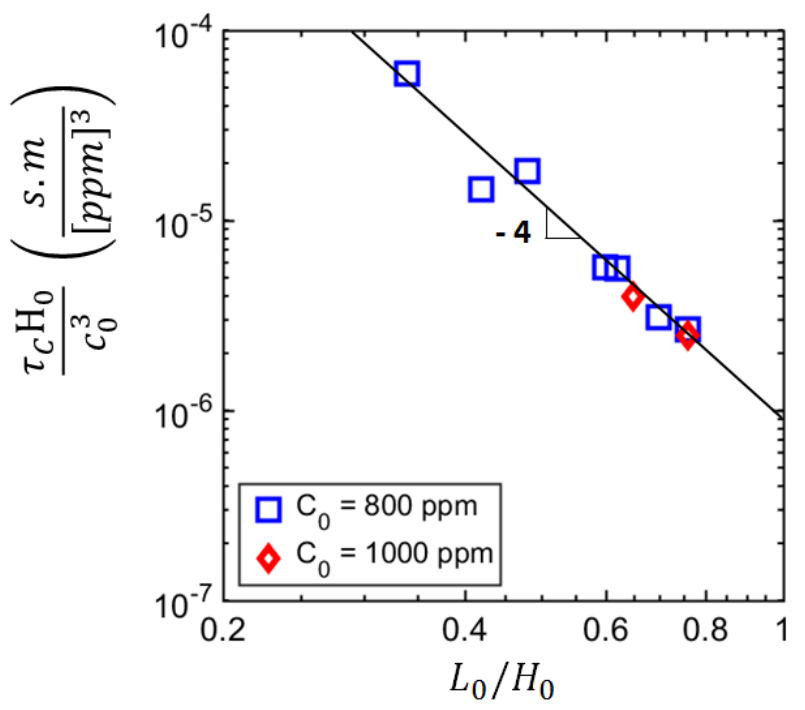

Fig. 5 Experimental plot of $\tau_{C} H_{0} / c_{0}^{3}$ as function of $L_{0} / H_{0}$ for different bottle tests at $800 \mathrm{ppm}$ and $1000 \mathrm{ppm}$ of Span 80

\subsection{Complete separation kinetics modelling of the dense zone}

In the previous section, we have seen qualitatively that the quasiplateau is the regime where the radius evolution is slow. In this section, we will show a complete model for the whole separation 
kinetics of the dense zone.

Firstly, we remark that the change of dense zone thickness is due to the coalescence of the water droplets in contact with the water phase interface. We will now write the rate of coalescence of water droplet with the water phase following the paths of the previous analysis. Let $S_{I}$ be the contact area of a droplet of radius $R_{i}$ at interface to the water phase. Let $L(t) \approx H_{0}-H_{w}$ is the thickness of dense zone, $L(0)=L_{0}$ being its initial value. The contact area $S_{I}$ can be estimated by the force balance (Figure 4):

$$
\Delta \rho g L \pi R_{i}^{2}=P_{f} S_{I}
$$

Where $P_{f}$ is the pressure inside the thin film. We assume that the surface contact is flat following 26 . Hence the pressure in the thin film is equal to the Laplace pressure:

$$
P_{f}=\frac{2 \gamma}{R_{i}}
$$

From equations (18) and (19) we deduce the contact area $S_{I}$ :

$$
S_{I}\left(R_{i}\right)=\frac{\pi \Delta \rho g L}{2 \gamma} R_{i}^{3}
$$

The coalescence of the last droplets to the water homophase induces a change of $L$. Each droplet of size R has the probability of coalescence $\omega S_{I}(R)$. Let $n_{S}\left(R_{i}\right)$ be the density per surface unit of droplets of radius $R_{i}$. The time evolution of this density is thus given

$$
d n_{S}\left(R_{i}\right)=-\omega n_{S}\left(R_{i}\right) S_{I}\left(R_{i}\right) d t
$$

When droplets coalesce with the water reservoir, the volume of each droplet is added to the water reservoir volume. This leads for $L$ to the following evolution :

$$
d L\left(R_{i}\right)=-d n_{S}\left(R_{i}\right) 4 \pi R_{i}^{3} / 3
$$

Combining the three previous equations, one gets

$$
\frac{d L}{d t}=\frac{2 \omega \pi^{2} \Delta \rho g L}{3 \gamma} \sum R_{i}^{6} n_{S}\left(R_{i}\right)
$$

where the sum corresponds to all droplets at the interfaces. To estimate this sum, let us remark that a droplet of radius $R_{i}$ occupies a surface area of $\pi R_{i}^{2}$. Thus if the density in number of the droplets of size $R_{i}$ near the interface is $\lambda_{S}\left(R_{i}\right)$, we have $n_{S}\left(R_{i}\right) \simeq \frac{\lambda_{S}\left(R_{i}\right)}{\pi R_{i}^{2}}$. We can thus rewrite the above equation:

$$
\frac{d L}{d t}=-\frac{2 \omega \pi \Delta \rho g L}{3 \gamma}\left\langle R_{i}^{4}\right\rangle
$$

where the bracket corresponds to the average over all droplets at the interfaces. We assume that the average of droplets size at interface evolves with time as already showed in the equation (12). Therefore the time evolution of dense zone thickness is described by:

$$
\frac{d L}{d t}=-\frac{A L}{\tau_{C}}\left(1-\frac{L}{L_{0}} \frac{t}{\tau_{C}}\right)^{-4 / 3}
$$

where $A=R_{0} / 3 L_{0}$. Equation (25) gives a complete time evolution of the separation kinetics of the dense zone. The solution of this equation exhibits 3 regimes as detailed in Appendix B:
- $t<\tau_{C}, L \simeq L_{0} e^{-A t / \tau_{C}}$ : Since $A<<1$ this evolution is very slow and mostly flat. This corresponds to the quasi-plateau as showed in the previous sections.

- $\tau_{C} \leq t<\tau_{C} / A$ : in that case $L(t)$ exhibits a simple scaling : $L(t) \simeq L_{0} \tau_{C} / t$. This evolution corresponds to the main part of the separation kinetics after the quasi-plateau.

- $\mathrm{t}>\tau_{C} / A$ : this last regime completes the separation of the dense zone. It has an asymptotic form: $L \simeq L_{0} \frac{\tau_{C}}{t} e^{-A t / \tau_{C}}$. Note that the time for observing this last regime is very long. One should wait at least at time of $3 L_{0} \tau_{C} / R_{0}$ to observe it. This time is larger by one or more order of magnitude than the quasi plateau duration.

Figure 6 below shows the experimental results of the 6 bottle tests that we have performed at various $L_{0}$ and $\tau_{C}$. The black straight line shows the complete numerical solution of equation (25) for $A<<1$. In our experiments, $A$ spans the range $1 / 3000$ to $1 / 1000$. The data points at the longest experimental times seem to deviate from the model. This is most likely due to the precision of these measurements, which is limited by the resolution of the images. Indeed, at these times the thicknesses measured are only a few pixels in height, $\sim 4$, and hence the relative incertitude for these measurements is high. Overall, the predictions of our model are in very good agreement with the experimental results, especially for the kinetics after the quasi-plateau where all the experimental points are likely to be unified into the model curve of $L(t) \simeq L_{0} \tau_{C} / t$.

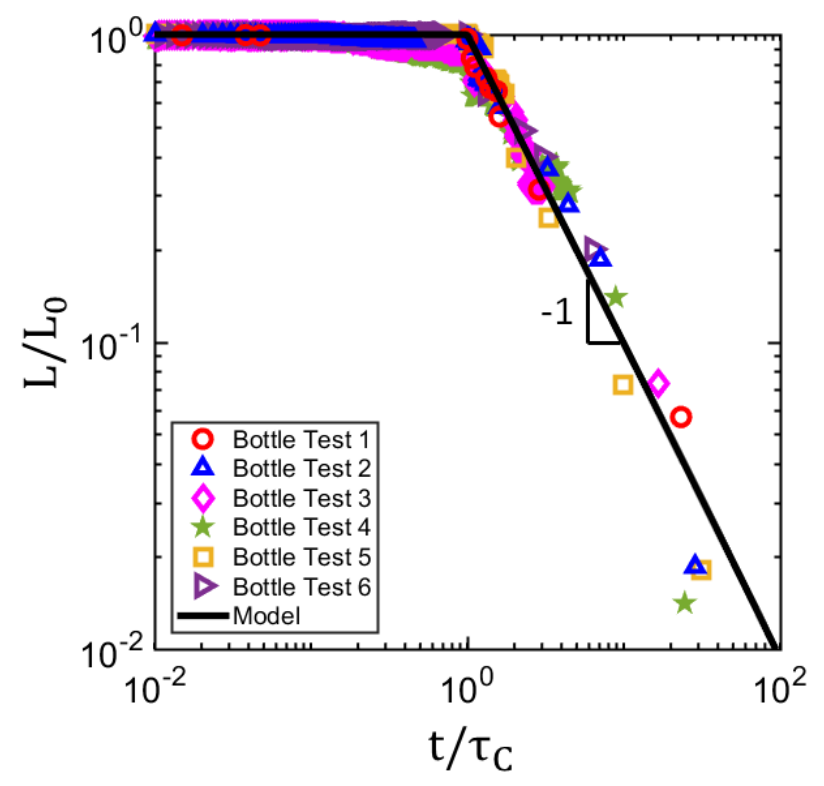

Fig. 6 Comparison between model and experimental data for different bottle tests at various Span 80 concentrations $c_{0}$ and mixing speed: (०) $c_{0}=800 \mathrm{ppm}$, U. Turrax $30000 \mathrm{RPM}(\triangle)(\diamond)(\star) c_{0}=800 \mathrm{ppm}$, U. Turrax 15000 RPM $(\square) c_{0}=600 \mathrm{ppm}$, U.Turrax $30000 \mathrm{RPM}$ and $(\triangleright) c_{0}=600$ ppm, U.Turrax 15000 RPM

\section{Conclusion}

To conclude, we have modelled the destabilisation of a surfactantstabilized concentrated emulsion submitted to gravity in the 
regime of thermally activated coalescence. The model is validated by our experiments. We deduce that the simple measure of the kinetics of an emulsion destabilisation can provide very relevant and quantitative measure of physico-chemical quantities. Firstly, after sedimentation, the height of the dense emulsion zone gives an information on the surfactant coverage of the droplets. Secondly, the time evolution of this dense zone is at the beginning extremely slow. It exhibits a quasi plateau whose duration gives the coalescence rate, $\omega$. After this plateau, a regime of fast coalescence is predicted and observed with a $1 / \mathrm{t}$ scaling for the dense zone thickness.

\section{Appendix A: Effect of polydispersity on droplets sur- face contact}

One may wonder how the polydispersity may affect colaescence. For that we can do the following estimation. First we count the number of neighbors of a droplet of radius $R_{i}$ surrounded by droplets of radius $R_{j}$. The solid angle occupied by a droplet $j$ at the periphery of the droplet $i$ is

$$
\sigma_{i j}=2 \pi\left(1-\cos \left(\arctan \frac{R_{j}}{R_{i}+R_{j}}\right)\right)
$$

Note that for $R_{i}=R_{j}$ the fraction of solid angle take a value of $\sigma_{i i} / 4 \pi$ and we know that in that case about 12 neighbors are surrounding a droplet. We get them for the compacity on the contacting neighbors a value of about $C_{\sigma}=3 \sigma_{i i} / 4 \pi$, and we deduce that the number of neighbors of radius $R_{j}$ around a droplets of radius $R_{i}$ can be estimated to

$$
Z_{i j}=\frac{4 \pi C_{\sigma}}{\sigma_{i j}}
$$

Thus expression 28 writes, for the droplet $i$

$$
\Delta \rho g z=\frac{Z_{i j}}{8 \pi R_{i}^{3}} \gamma\left(\frac{1}{R_{i}}+\frac{1}{R_{j}}\right) S_{i j}\left(R_{i}+R_{j}\right)
$$

Thus, the surface contact of droplet of radius $R_{i}$ surrounded by droplets of ridus $R_{j}$ writes :

$$
S_{i j}=\Delta \rho g z C_{\sigma} \frac{R_{i}^{3} \sigma_{i j}}{\gamma\left(\frac{1}{R_{i}}+\frac{1}{R_{j}}\right)\left(R_{i}+R_{j}\right)}
$$

Note that $S_{i j}$ is maximum when $R_{i} \simeq R_{j}$, so the polydispersity may not be an important issue.

\section{Appendix B: Analyze of the solution of equation for separation kinetics}

Let $\tilde{L}=L / L_{0}, T=t / \tau_{C}$ and $A=R_{0} / 3 L_{0}$, we thus can rewrite the equation (25) in dimensionless form:

$$
\frac{d \tilde{L}}{d T}=-A \tilde{L}(1-\tilde{L} T)^{-4 / 3}
$$

with the initial condition $\tilde{L}(0)=1$. Note also that $A<<1$

- If $\mathrm{T}$ is small, $1-\tilde{L} T \approx 1$ so $\tilde{L} \approx e^{-A T}$. This regime is no longer valid when $\mathrm{T}$ approaches 1 .
- If $T \geq 1$, an asymptotic solution for large $\mathrm{T}$ is

$$
\tilde{L} \simeq \frac{e^{-A T}}{T}
$$

In fact, a numerical solution of equation (30) shows that this solution is a good approximation when $\mathrm{T}$ is larger than 1 , as shown in figure for $A=0.001$. As long as $A T<<1, \tilde{L}$ can be approximated by

$$
\tilde{L} \simeq \frac{1}{T}
$$

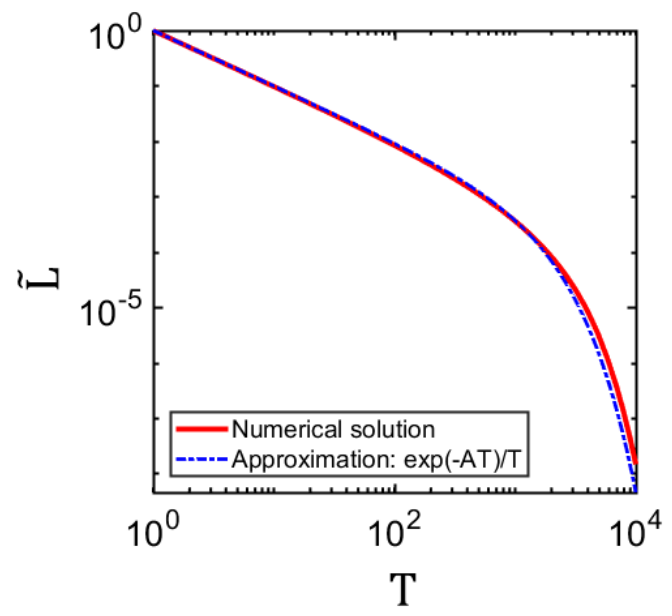

Fig. 7 Numerical solution of equation 30 and its approximation

\section{Nomenclature}

$\Delta \rho \quad$ Density difference of aqueous and oil phase

$\Gamma_{\text {sat }} \quad$ Saturated surface excess concentration of surfactant

$\omega \quad$ Coalescence frequency per unit area

$\Phi \quad$ Water volume fraction in the emulsion system

$\tau_{C} \quad$ Length of the quasi-plateau in unit of time

$c^{\star} \quad$ Minimum surfactant concentration to generate the dense emulsion zone

$c_{0} \quad$ Total surfactant concentration

$H_{0} \quad$ Height of water column prior to emulsification

$H_{w}^{\star} \quad$ Position of the quasi-plateau

$L \quad$ Thickness of the dense emulsion zone

$L_{0} \quad$ Initial thickness of the dense emulsion zone, at the beginning of the second regime

$R_{0} \quad$ Mean droplet radius of emulsion

$\gamma \quad$ Water/oil interfacial tension

\section{Conflicts of interest}

There are no conflicts of interest to declare. 


\section{Acknowledgements}

We acknowledge Total S.A. management for giving the permission to publish this work. We thank Maurice Bourrel, Nicolas PassadeBoupat, and Thierry Palermo for fruitful discussions.

\section{References}

1 Coalescence in foams and emulsions | Encyclopedia of Surface and Colloid Sciences, Taylor \& Francis, London 2012, 2012.

2 E. Basheva, T. Gurkov, I. Ivanov, G. Bantchev, B. Campbell and R. Borwankar, Langmuir, 1999, 15, 6764-6769.

3 N. I. Politova, S. Tcholakova, S. Tsibranska, N. D. Denkov and K. Muelheims, Colloids and Surfaces A, 2017, 531, 32-39.

4 K. Khristov, D. Exerowa and G. Minkov, Colloids Surf. A, 2002, 210, 159.

5 S. Tcholakova, N. D. Denkov and A. Lips, Phys. Chem. Chem. Phys., 2007, 10, 1608.

6 D. Georgieva, V. Schmitt, F. Leal-Calderon and D. Langevin, Langmuir, 2009, 25(10), 5565-5573.

7 G. G. Fuller and J. Vermant, Annual Review of Chemical and Biomolecular Engineering, 2012, 3, 519-543.

8 J. Maldonado-Valderrama, A. Martin-Molina, A. MartinRodriguez, M. Cabrerizo-Vilchez, M. Galvez-Ruiz and D. Langevin, Journal of Physical Chemistry C, 2007, 111, 2715-2723.

9 A. Kabalnov and H. Wennerstrom, Langmuir, 1996, 12, 276292.

10 A. de Vries, J. Recl. Trav. Chim. Pays-Bas, 1958, 77, 383,.

11 V. Schmitt and F. Leal-Calderon, Europhys. Lett., 2004, 67(4),
$662-668$.

12 A. Hasmy, R. Paredes, O. Sonneville-Aubrun, B. Cabane and R. Botet, Phys. Rev. Lett., 2000, 82, 3368.

13 L. R. D. Frenkel, J. Chem. Phys., 2007, 127, 134701.

14 E. Forel, B. Dollet, D. Langevin and E. Rio, Phys. Rev. Lett., 2019, 122, 088002(5).

15 T. Krebs, C.G.P.H.Schroën and R.M.Boom, Chemical EngineeringScience, 2012, 71, 118-125.

16 S. Tcholakova, N. Denkov, I. Ivanov and B. Campbell, Langmuir, 2002, 18, 8960-8971.

17 G. A. van Aken and F. D. Zoet, Langmuir, 2000, 16, 71317138.

18 S. Jeelani and S. Hartland, AIChE J., 1985, 31, 711-720.

19 C. Noïk, T. Palermo and C. Dalmazzone, Journal of Dispersion Science and Technology, 2013, 34, 1029-1042.

20 K. Roger and B. Cabane, Angew. Chem., Int. Ed., 2012, 51, 5625-5628.

21 Emulsion Science | Basic Principles. An Overview, Srpinger, 2002.

22 B. Riechers, F. Maes, E. Akoury, B. Semin, P. Gruner and J.-C. Baret, PNAS, 2016, 113 (41), 11465-11470.

23 T. Krebs, D. Ershov, C. G. P. H. Schroen and R. M. Boom, Soft Matter, 2013, 9, 4026-4035.

24 H. Princen, J. Colloid Interface Sci., 1983, 91, 160-175.

25 J. Brujic, S. F. Edwards, D. V. Grinev, I. Hopkinson, D. Brujic and H. A. Makse, Faraday Discussion, 2003, 123, 207-220.

26 H. Feng, D. Ershov, T. Krebs, K. Schroen, M. A. C. Stuart, J. van der Gucht and J. Sprakel, Lab Chip, 2015, 15, 188194. 